EPJ Web of Conferences 60, 20015 (2013)

DOI: $10.1051 /$ epjconf $/ 20136020015$

(C) Owned by the authors, published by EDP Sciences, 2013

\title{
Measurement of top quark polarisation with the ATLAS experiment
}

\author{
Sara Borroni ${ }^{1, a}$ on behalf of the ATLAS Collaboration \\ ${ }^{1}$ Deutsches Elektronen-Synchroton, DESY, Notkestraße 85, D-22603 Hamburg, Germany
}

\begin{abstract}
While in the Standard Model top quarks are predicted to be produced unpolarized at the LHC, several models beyond the SM predict a non-zero polarisation. In particular, some of the models predicting a larger forward-backward $t \bar{t}$ asymmetry than the SM, as measured at the Tevatron, predict a positive top quark polarisation. Using $4.66 \mathrm{fb}^{-1}$ of data collected by the ATLAS experiment at a centre of mass energy of $7 \mathrm{TeV}$, a measurement of the top quark polarisation is presented. The full $t \bar{t}$ system is reconstructed from final states with at least one charged lepton, and a template fit to the distribution of the angle between the charged lepton and the top quark is performed to measure the top quark polarisation.
\end{abstract}

In the Standard Model (SM) top quarks in top-antitop $(t \bar{t})$ production are predicted to be produced unpolarized in proton-proton collisions at the LHC due to parity conservation and an unpolarized initial state. However, several models beyond the SM predict a non-zero polarisation [1-3], and among these some even predicts a larger forward-backward top-antitop asymmetry than the SM, as measured at the Tevatron [4-6].

Here a measurement of the top quark polarisation in single lepton $t \bar{t}$ events $(t \bar{t} \rightarrow l v q \bar{q} b \bar{b})$ is presented [7]. The analysis is performed using the full 2011 dataset of $p p$ collisions, collected by the ATLAS experiment at a center of mass energy of $7 \mathrm{TeV}$, corresponding to an integrated luminosity of $4.66 \pm 0.8 \mathrm{fb}^{-1}$.

Since the top quark decays before hadronising, its spin information is conserved, and can be extracted from the distribution of the polar angle, $\theta_{i}$, of any of its final state decay products $i$. Such a distribution with respect to a given quantisation axis is given by:

$$
W\left(\cos \theta_{i}\right) \propto \frac{1}{2}\left(1+\alpha_{i} P \cos \theta_{i}\right),
$$

where $P$ is the degree of polarisation along this axis and $\alpha_{i}$ is the spin analysing power of the final state particle considered. At the tree level, charged leptons $(\ell)$ are predicted to have the largest sensitivity to the parent top quark polarisation, having $\alpha_{\ell}=1$. Therefore, the $\cos \theta_{\ell}$ distribution of the charged leptons is used as the observable for this measurement, the quantisation axis being chosen as the top quark's momentum direction in the $t \bar{t}$ center of mass frame.

Single lepton $t \bar{t}$ events are selected by requiring:

- Exactly one high- $p_{\mathrm{T}}{ }^{1}$, isolated electron or muon. This lepton should have fired a single lepton trigger. No at-

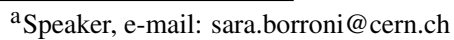

${ }^{1}$ ATLAS uses a right-handed coordinate system with its origin at the nominal interaction point (IP) in the center of the detector and the $z$-axis
}

tempt is made to remove leptons originating from $\tau$ decays, which are considered part of the signal,

- At least four well reconstructed jets, out of which at least one is tagged as originating from a $b$-quark by a multivariate algorithm at the $70 \%$ efficiency working point,

- Large missing transverse momentum $\left(E_{\mathrm{T}}^{\mathrm{miss}}\right)$ from the neutrino escaping the detector,

- High transverse mass of the leptonically decaying $W$ boson, computed from the lepton $p_{\mathrm{T}, \ell}$ and $\phi_{\ell}$ angle and the $E_{\mathrm{T}}^{\mathrm{miss}}$ as $m_{\mathrm{T}}=\sqrt{2 p_{\mathrm{T}, \ell} E_{\mathrm{T}}^{\mathrm{miss}}\left[1-\cos \left(\phi_{\ell}-\phi\left(E_{\mathrm{T}}^{\mathrm{miss}}\right)\right)\right]}$.

Two channels, referred to as the electron and muon channel based on the flavour of the selected lepton, are considered. They are analysed separately and then combined.

The SM expectations for the various distributions are obtained from Monte Carlo (MC) simulated samples. Next-to-leading order event generators are used to simulate $t \bar{t}$, single top quark and diboson events. Leading order plus extra-legs generators are used for $Z+$ jets events. The MC expectations are then rescaled to the most upto-date available theoretical computations. The $W+$ jets events contribution is also estimated using MC simulation, but the number of expected events is then rescaled to match the most recent measurement of the top-antitop production charge asymmetry [8]. Multijet events can contaminate the signal region when one jet is mis-identified as a lepton. This contribution is estimated using a data-driven matrix method. The number of jets passing the full tight lepton selection is computed from the number of jets passing a looser lepton selection and applying a loose-to-tight

along the beam pipe. The $x$-axis points from the IP to the center of the LHC ring, and the $y$ axis points upward. Cylindrical coordinates $(R, \phi)$ are used in the transverse plane, $\phi$ being the azimuthal angle around the beam pipe. The pseudorapidity is defined in terms of the polar angle $\theta$ as $\eta=-\ln \tan (\theta / 2)$. The transverse energy $E_{\mathrm{T}}$ is defined as $E \sin \theta$, where $E$ is the energy associated to the calorimeter cell or energy cluster. Similarly, $p_{\mathrm{T}}$ is the momentum component transverse to the beam line. 
ratio which is independently measured in a dedicated orthogonal data sample.

Selected events need to be reconstructed in order to determine the quantisation axis, i.e. the four-momenta of the top and antitop quarks, and to compute the $\cos \theta_{\ell}$ angle. A likelihood fit, already used for the top quark mass measurement determination as described in Ref. [9], is used to determine the neutrino momentum and the assignment of final state jets to the top and antitop quark decays. Figure 1 shows the distribution of the reconstructed $p_{\mathrm{T}}$ of the $b$-quark jet associated with the leptonically decaying $W$ boson, and the longitudinal momentum of the reconstructed neutrino. Data and MC expectations for signal and background are found to be in good agreement for the reconstructed $\cos \theta_{\ell}$ distributions as shown in figure 2 both for the electron and muon channels.

To measure the polarisation of the top quarks, a template fit to the reconstructed $\cos \theta_{\ell}$ distribution of signal and background is performed. Since no dedicated MC samples with polarised top quarks are available, signal templates with fully positively and negatively polarised $(P= \pm 1)$ top quarks are created from the default sample. The reconstructed level $\cos \theta_{\ell}$ distribution of all $t \bar{t}$ events is reweighted with $\left(1+\cos \theta_{\ell, \text { gen }}\right)$ and $\left(1-\cos \theta_{\ell, \text { gen }}\right)$, respectively, being $\theta_{\ell \text {,gen }}$ the generated value of the $\theta_{\ell}$ angle from MC simulation, to build the positive and the negative templates to be used in the fit.

The result of the fit is the fraction assigned to the template for positively polarised top quark $f$, which is related to the polarisation at the generator level via the equation:

$$
\begin{aligned}
\frac{1}{2}\left(1+\alpha_{\ell} P \cos \theta_{\ell, \text { gen }}\right)= & \frac{1}{2} f\left(1+\cos \theta_{\ell, \text { gen }}\right) \\
& +\frac{1}{2}(1-f)\left(1-\cos \theta_{\ell, \text { gen }}\right)
\end{aligned}
$$

The template fit is performed for each channel separately and the likelihoods are combined for the final result. All correlations between the channels are taken into account. The $t \bar{t}$ production cross section is simultaneously fitted to reduce the impact of the normalization uncertainty on the fit result. It is found to be in agreement with the SM expectation. Figure 3 shows the templates for positively and negatively polarised top quarks, as well as the fit result for the electron and muon channels separately.

The combined result is:

$$
f=0.470 \pm 0.009(\text { stat })_{-0.032}^{+0.023} \text { (syst) }
$$

which translates to:

$$
\alpha_{\ell} P=-0.060 \pm 0.018(\text { stat })_{-0.064}^{+0.046}(\text { syst }) .
$$

The dominant experimental systematic uncertainty on this measurement is due to uncertainty on the jet energy scale and resolution. The dominant theoretical uncertainty is due to the top quark mass, estimated taking into account the variation of $f$ when the mass is varied by \pm 1 $\mathrm{GeV}$. Other signal modelling uncertainties considered include the effect of the generator used to model the signal, the description of the initial and final state radiation, color reconnection and the modelling of fragmentation, as reported in table 1 .

Concluding, a measurement of the top quark polarisation in $t \bar{t}$ events has been performed, using the $4.66 \mathrm{fb}^{-1}$ data sample collected by the ATLAS experiment in $7 \mathrm{TeV}$ center of mass energy $p p$ collisions. The result has been found to be in agreement with the SM predictions.

Table 1. Summary of the systematic uncertainties on $f$ in the single lepton final states [7]. The jet reconstruction is dominated by the energy scale uncertainty, while the signal modeling is dominated by the uncertainty in the top quark mass.

\begin{tabular}{ccc}
\hline Source & \multicolumn{2}{c}{$\Delta f$} \\
\hline Lepton reconstruction & +0.002 & -0.003 \\
Jet reconstruction & +0.018 & -0.028 \\
$E_{\mathrm{T}}^{\text {miss }}$ reconstruction & +0.001 & -0.003 \\
Signal modelling & +0.011 & -0.012 \\
$W+$ jets shape & +0.004 & -0.004 \\
Fake lepton shape & +0.004 & -0.005 \\
MC background cross section & +0.002 & -0.002 \\
Template statistical uncertainty & +0.004 & -0.004 \\
\hline Total systematic & +0.023 & -0.032 \\
\hline \hline
\end{tabular}

\section{References}

[1] D. Krohn, T. Liu, J. Shelton and L. -T. Wang, Phys. Rev. D 84 (2011) 074034

[2] S. Fajfer, J. F. Kamenik and B. Melic, JHEP 1208 (2012) 114

[3] J. A. Aguilar-Saavedra and M. Perez-Victoria, arXiv:1302.6618.

[4] V. M. Abazov et al. (D0 Collaboration), Phys. Rev. D 84 (2011) 112005

[5] V. M. Abazov et al. (D0 Collaboration), arXiv:1207.0364.

[6] T. Aaltonen et al. (CDF Collaboration), Phys. Rev. D 87 (2013) 092002

[7] ATLAS Collaboration, ATLAS-CONF-2012-133 https://cds.cern.ch/record/1478373

[8] ATLAS Collaboration, Eur. Phys. J. C 72 (2012) 2039

[9] ATLAS Collaboration, Eur. Phys. J. C 72 (2012) 2046 

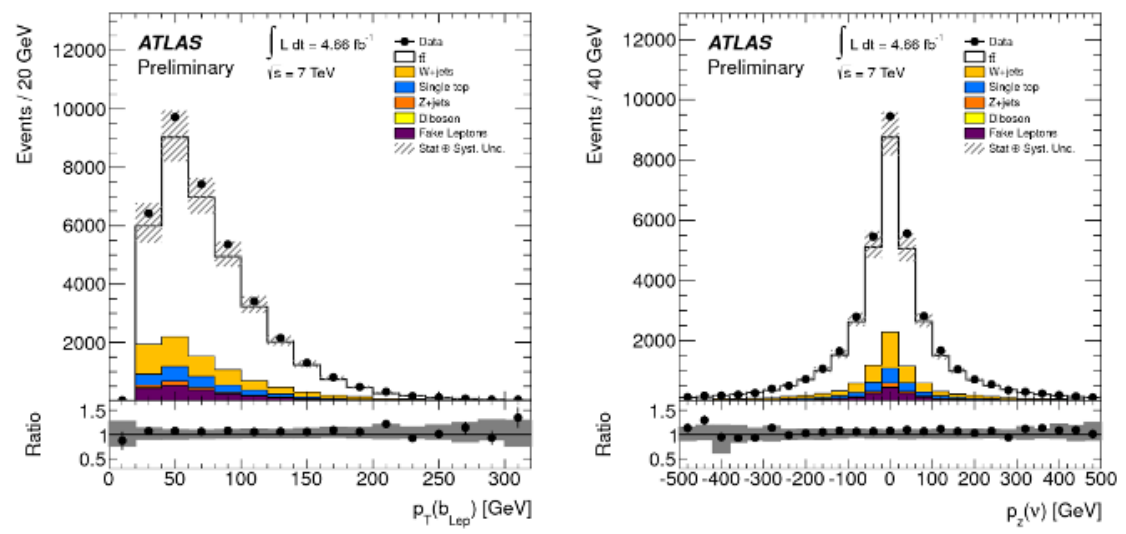

Figure 1. Transverse momentum distribution of the $b$-quark jet associated to leptonically decaying $W$ boson (left), and the longitudinal momentum of the neutrino (right), after the event reconstruction [7]. Data and MC signal and background expectations are compared, and the total systematic and statistical uncertainty on the expectations is shown by the shaded area.
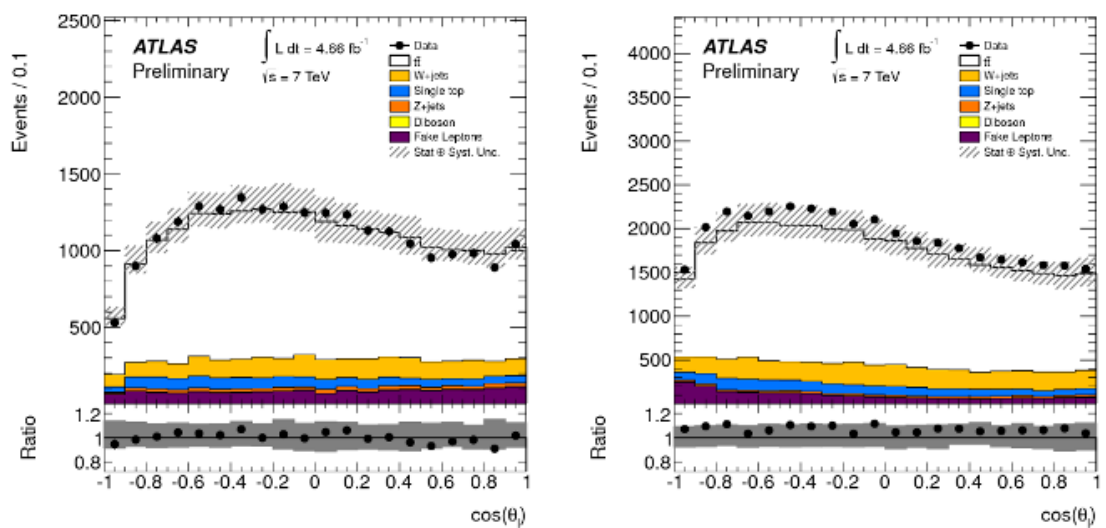

Figure 2. Distribution of $\cos \theta_{\ell}$ of the charged leptons for the electron and muon channel (left and right, respectively) [7]. Data and MC signal and background expectations are compared, and the total systematic and statistical uncertainty on the expectations is shown by the shaded area.
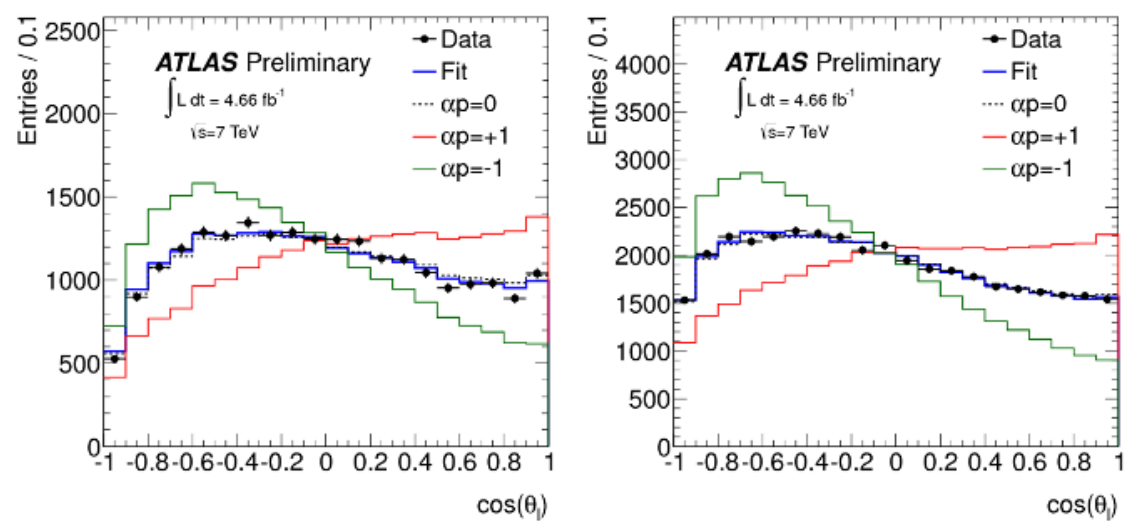

Figure 3. Fitted $\cos \theta_{\ell}$ distribution (blue line) for the electron and muon channels (left and right, respectively) [7]. The templates corresponding to positively (red line), negatively (green line) and non-polarised (blue dashed line) top quarks are also shown. 